\title{
Mild cognitive impairment, risk of depression, quality of life and frailty in elderly users of Primary Care, Chile.
}

\author{
Authors: Cristóbal Heskia1, Daniela Urrutia ${ }^{1}$, Jorge Santander ${ }^{1}$, Rodrigo Aguirre ${ }^{2}$, Alexander Wenner ${ }^{1}$ \\ 1: Instituto de Neurociencias Clínicas, Facultad de Medicina, Universidad Austral de Chile. \\ 2: Instituto de Estudios Psicológicos, Facultad de Medicina, Universidad Austral de Chile. \\ This investigation is supported by Vicerrectoría de Investigación, Desarrollo y Creación Artística (VIDCA) of Universidad Austral de Chile. Acknowledgments: CESFAM Jorge Sabat, CESFAM Las Animas and \\ Departamento de Salud Mental Municipal (DESAM) of Valdivia, Chile. Informed consent approved by Comité de Ética, Servicio de Salud Valdivia.
}

Background: Alzheimer's disease (AD) is preceded by Minor cognitive impairment $(\mathrm{MCl})$, which is correlated with risk factors such as frailty, polypharmacy, geriatric depression (GD), loneliness, low quality of life, among others. Studies suggest that controlling risk factors and the early detection of $\mathrm{MCl}$ is key for $A D$ prevention $(1,2)$. In Chile, the use of screening tools for cognitive impairment in clinical practice is scarce. Therefore, in the effort against $A D$, it is important to scope geriatric populations for $\mathrm{MCl}$ and its risk factors on a local scale. Objectives: to determine the cognitive performance, risk of depression, quality of life (QOL), frailty, polypharmacy and dependence in elderly primary care users in Valdivia, Chile. Methods: a descriptive observational design, consisting in a questionnaire including WHOQOL-BREF for QOL, Frail test for frailty, Geriatric Depression Scale (GDS-15 or Yesavage) and Pfeffer Functional Activities Questionnaire (PFAQ) for autonomy vs dependence; and the application of the Montreal Cognitive Assessment test (MoCA) for cognitive performance by trained interviewers to adults over the age of 60 able to read, write, give consent and without visual or auditive impairment, selected in two primary care centre waiting rooms with a systematical sampling. The data obtained is analyzed via Epiinfo. Main outcomes: "Subjective memory complaints (SMC)"; "MCl": MoCA = 20 pts.; "MCl or other cognitive impairment": MoCA $\leq 20$ pts.(3); "Risk of GD": GDS-15 $\geq 6$ pts. (4); "Frailty": Frail $\geq 3$ pts.; "Pre-frailty": Frail = 1-2 pts. (5); "Polypharmacy": $\geq 5$ drugs (6); "Dependence": PFAQ $\geq 6$ pts. (7); and "QOL" including the following health dimensions: physical, psychological, social relationships and environment (8).

Figure 1: Risk of geriatric depression.

Normal Mild depression Moderate to severe depression

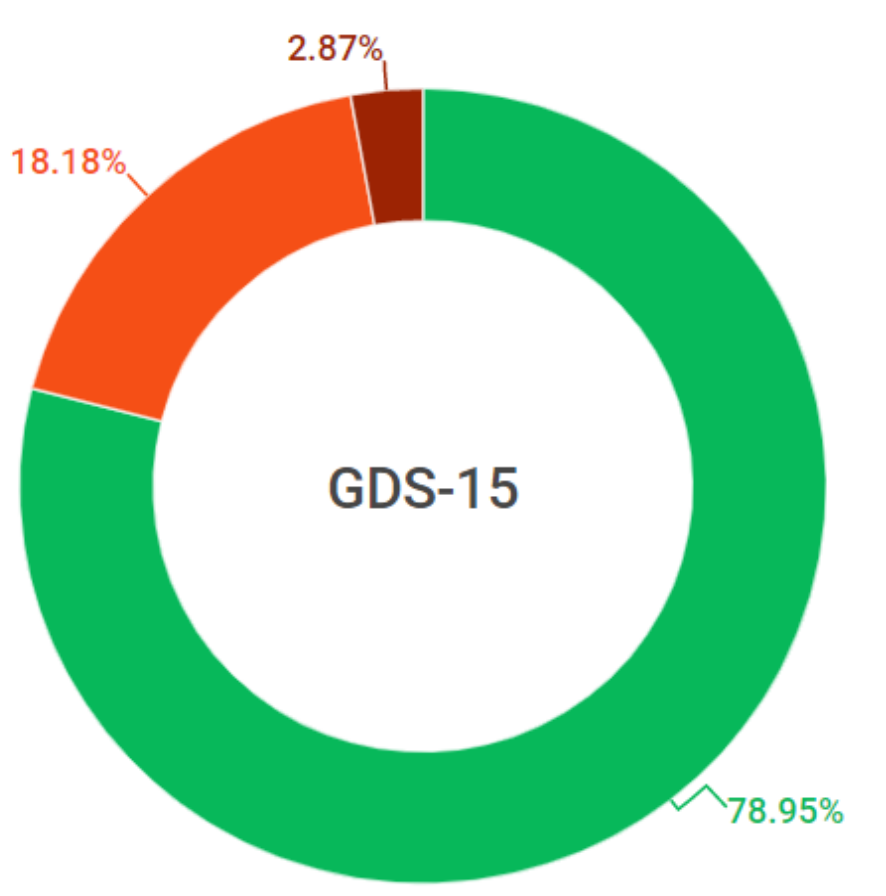

Results and conclusion: In total, 209 older adults between the ages of 60 and 89 years were surveyed, with a frequency of $39,2 \%$ for SMC, $12,9 \%$ for $\mathrm{MCl}$ and $49,3 \%$ for "MCl or other cognitive impairment". Demographic data, cognitive performance and polypharmacy are illustrated in the table \#1. About the factors related to cognitive impairment, $21,1 \%$ were at risk of GD (Figure \#1), 48,8\% met criteria for pre-frailty and 19,6\% for frailty (Figure \#2), 34,9\% for polypharmacy and 3,3\% for dependence (Figure \#3). The dimensions of QOL are illustrated in the figure \#4. These results, including a majority of older adults scoring positive for $\mathrm{MCl}$ or probable mild dementia, suggest an urgent need in our local community for further screening.

\begin{tabular}{|c|c|c|c|}
\hline Mean age, years (SD) & $70,9(7,3)$ & & \\
\hline Female gender, $n(\%)$ & $144(68,9)$ & & \\
\hline Married or living with a partner, $\mathrm{n}(\%)$ & $29(13,9)$ & & \\
\hline \multicolumn{4}{|l|}{ Education, $\mathrm{n}(\%)$} \\
\hline None & $4(1,9)$ & & \\
\hline Basic education & $108(51,7)$ & & \\
\hline Middle education & $67(32,1)$ & & \\
\hline University or technical education & $30(14,4)$ & & \\
\hline SMC, n (\%) & $82(39,2)$ & & \\
\hline \multicolumn{4}{|l|}{ MoCA } \\
\hline Normal; n (\%) & $106(50,7)$ & & \\
\hline $\mathrm{MCl} ; \mathrm{n}(\%)$ & $27(12,9)$ & & \\
\hline "Other cognitive impairment"; n (\%) & $103(49,3)$ & & \\
\hline Cognitive performance by dimensions: & Mean (SD) & Min & Max \\
\hline Visuospatial/executive & $2,6(1,4)$ & 0 & 5 \\
\hline Identification & $2,6(0,7)$ & 0 & 3 \\
\hline Attention & $3,7(1,6)$ & 0 & 6 \\
\hline Language & $1,8(1)$ & 0 & 3 \\
\hline Abstraction & $1,1(0,8)$ & 0 & 2 \\
\hline Memory & $1,7(1,6)$ & 0 & 5 \\
\hline Orientation & $5,8(0,5)$ & 2 & 6 \\
\hline Polypharmacy, $n$ (\%) & \multicolumn{3}{|l|}{$73(34,9)$} \\
\hline
\end{tabular}

Figure 2: Frialty (Frail test).

Figure 3: Functionality.

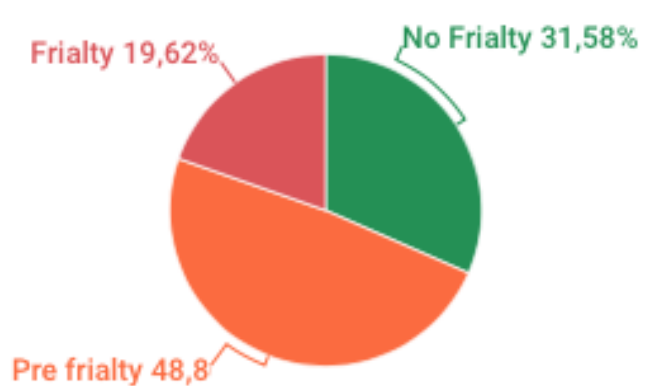

Autonomy Dependence

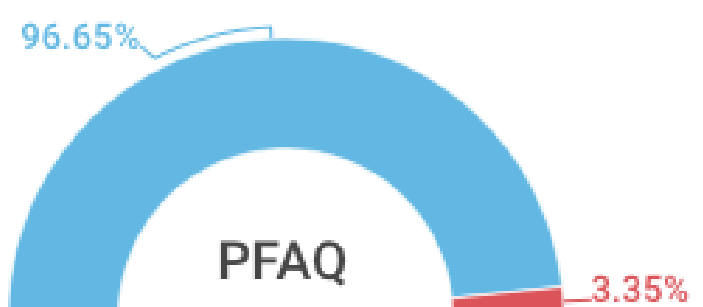

Figure 4: Quality of life.

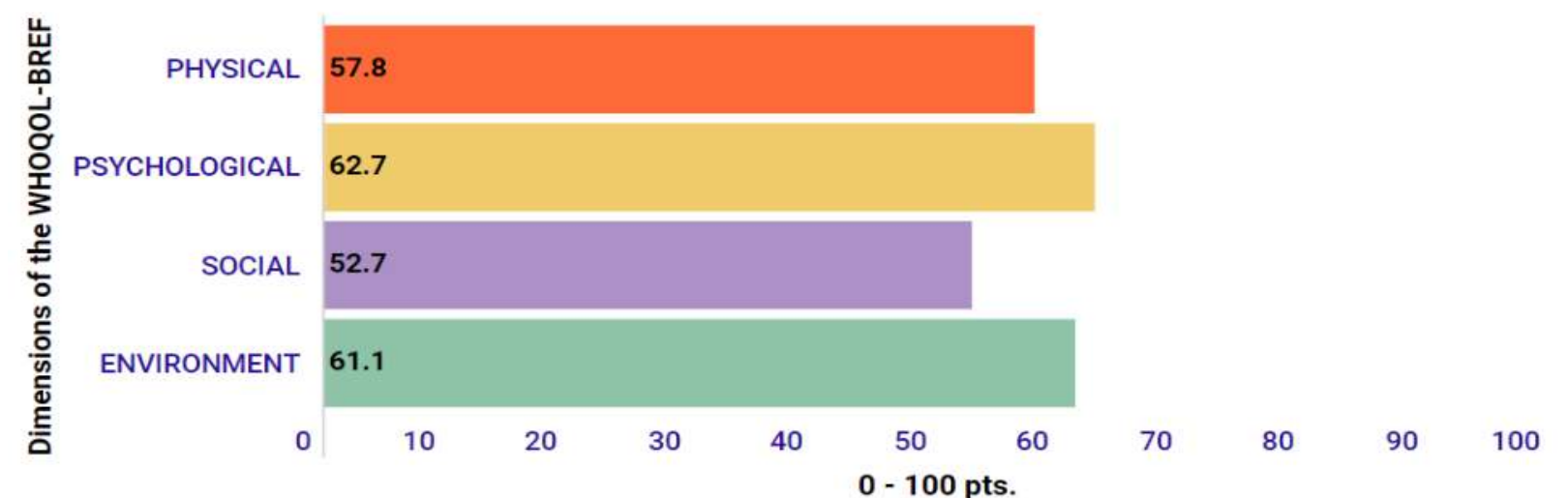

